\title{
Pictogramas y adultos mayores: señalética de un sistema de transporte masivo en Colombia ${ }^{1}$
}

\author{
Pictograms and elderly persons: wayfinding signage \\ of a Colombian Bus Rapid Transit
}

Cómo citar:

Romo, G \& Marulanda, J. (2020). Pictogramas y adultos mayores: señalética de un sistema de transporte masivo en Colombia. Designia 7(2), 17-39.

\footnotetext{
${ }^{1}$ Artículo que presenta los resultados de del trabajo de grado titulado

“Interpretación de los pictogramas de la señalética del MIO por parte de los ciudadanos de la tercera edad en la ciudad de Santiago de

Cali" para optar al título de diseñadora de la comunicación gráfica.

* Estudiante de Diseño de la Comunicación Gráfica de la Universidad Autónoma de Occidente. Obtuvo el mejor puntaje en las pruebas Saber Pro 2018.

E-mail: gwendyromovaldes@gmail.com ORCID: https://orcid.org/0000-0002-6551-5668

**Docente de Diseño de la Universidad Autónoma de Occidente. E-mail: jmarulanda@uao.edu.co ORCID: https://orcid.org/0000-0003-3984-1803
}

\section{Palabras clave:}

Señalética, pictogramas, adulto mayor, sistema de transporte masivo.

\section{Key words:}

Wayfinding signage, pictograms, elderly-user, Bus Rapid Transit (BRT).

Recibido: 05/06/2019 Aceptado: 06/09/2019

\section{Resumen:}

El presente artículo presenta los resultados del trabajo de grado titulado "Interpretación de los pictogramas de la señalética del MIO por parte de los ciudadanos de la tercera edad en la ciudad de Santiago de Cali" para optar por el título de diseñadora de la comunicación gráfica. La investigación tiene como objetivo investigar la comprensión de 38 pictogramas de la señalética para un sistema de transporte masivo del suroccidente de Colombia. Se exploró la comprensión de pictogramas siguiendo la siguiente metodología: i) realización de tres entrevistas a funcionarios-administrativos del sistema de transporte, con conocimiento sobre las políticas institucionales asociadas 
con el desarrollo de señalética (dos funcionarios del programa para adultos mayores, y un funcionario de la dirección comercial y servicio al cliente); ii) desarrollo y aplicación de un cuestionario para 72 personas mayores de 60 años, distribuidas en dos grupos equitativos (usuarios y no usuarios del sistema), para explorar la comprensión de 38 pictogramas (ilustrados) de buses, estaciones y terminales; iii) desarrollo y aplicación de un nuevo cuestionario para ocho adultos mayores usuarios del sistema, a quienes se les presentaron 13 pictogramas (fotografiados in situ) que fueron de difícil comprensión en el cuestionario anterior. Los resultados muestran que dichos pictogramas pueden ser de mejor acceso y comprensión cuando se ponen en contexto (fotografiados in situ). Este estudio confirma la importancia de evaluar la comprensión de la señalética en contextos situados (condiciones físicas) que incluyan características de las audiencias usuarias (nivel educativo, grupos etarios, saberes culturales y género).

\section{Abstract:}

This paper is based on a senior thesis in Graphic Design. The study aims at exploring the comprehension level for 38 wayfinding pictograms of a Southwest Bus Rapid Transit (BRT) in Colombia. The research design as follows: i) interviewing three staff members of the BRT as key informants regarding wayfinding institucional policies (two staff from elderly program, and one affiliated to management and customer service); ii) designing and applying a questionnaire for 72 senior citizens older than 60 years in two equal groups (users and non-users) to explore the comprehension level for 38 illustrated wayfinding pictograms of buses, bus stations, and terminals; iii) designing and applying another questionnaire for eight senior citizens to explore the comprehension level for 13 wayfinding pictograms pictured in situ, which were rated as hardly understood by the BRT elderly users. The findings show that pictogram comprehension is improved when wayfinding pictogram is presented in situ. This study also confirms that evaluating user comprehension of wayfinding pictogram might consider situated contexts (physical location) and sociodemographic user features (educational level, age groups, cultural background, and gender). 


\section{INTRODUCCIÓN}

En la región latinoamericana se han implementado 54 sistemas de tipo BRT (bus rapid transit, traducido como sistema de transporte masivo) que movilizan el 60.74\% de los usuarios de este tipo de transporte en el mundo (BRTData.org, citado en Ojeda, 2018). Dado el volumen de población movilizada, puede esperarse que los usuarios de sistemas de transporte masivo se enfrenten, en ocasiones, con dificultades de uso. Por ejemplo, para el caso del BRT de la capital colombiana, un estudio reportó que el $72 \%$ de los encuestados (385 personas en total) perciben como poco seguro el sistema, y solo el 30\% están satisfechos con la frecuencia con la que pasan los buses (Vega, Rivera \& Malaver, 2017). En este panorama, los adultos mayores son etiquetados como un usuario común, carentes de mayores privilegios que reconozcan sus condiciones físicas y mentales. En la vejez disminuye la atención voluntaria, afectando la detección de señales y el grado de vigilancia; también, se afecta la atención selectiva para percibir y discriminar estímulos (Sánchez y Pérez, 2008). En efecto, Lámbarry, Rivas y Trujillo (2013, p. 90), en un estudio sobre percepción de la calidad del servicio de BRT, sugieren en ese sentido para futuras investigaciones identificar patrones diferentes de los usuarios, según segmentos de mercado. 
Atendiendo a lo anterior, el presente artículo surge de un trabajo de grado que tuvo como objetivo conocer la comprensión de 38 pictogramas de la señalética para un sistema de transporte masivo del suroccidente de Colombia. Para plantearlo, se realizó una observación exploratoria en 10 estacionamientos que hizo visible las condiciones de interacción del adulto mayor con la señalética del sistema, entre las que se pueden mencionar:

- Falta de comprensión por parte del usuario, que termina siendo subsanada por la interacción personal con los funcionarios.

- Pérdida en la localización por falta de comprensión del cronograma de rutas y de estrategias de abordaje.

- Problemas con el uso del sistema de pago con tarjeta electrónica.

- Pérdida de tiempo al usar el servicio.

- Dificultad con el uso de servicios preferenciales (Figura 1).

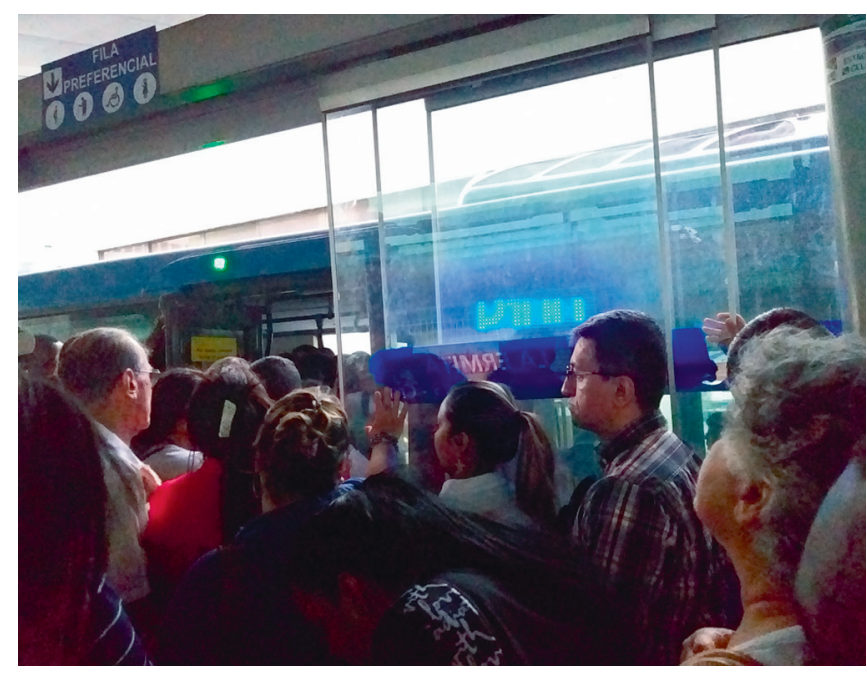

Figura 1. Ausencia de fila preferencial, a pesar de que entre los usuarios hay adultos mayores.

Fuente: Gwendy Romo.

El trabajo de investigación se plantea someter la pictografía del sistema masivo de transporte a una evaluación por parte de usuarios adultos mayores, quienes confrontarán su funcionalidad "sin la necesidad de conocer numerosas pautas convencionales para la comprensión" (Beltrán, Durán, Figueroa, Gutiérrez y Zuleta, 2015). A continuación, se observan algunas aplicaciones de dicha pictografía, con dos versiones diferentes del cada pictograma (Figuras 2, 3 y 4): 

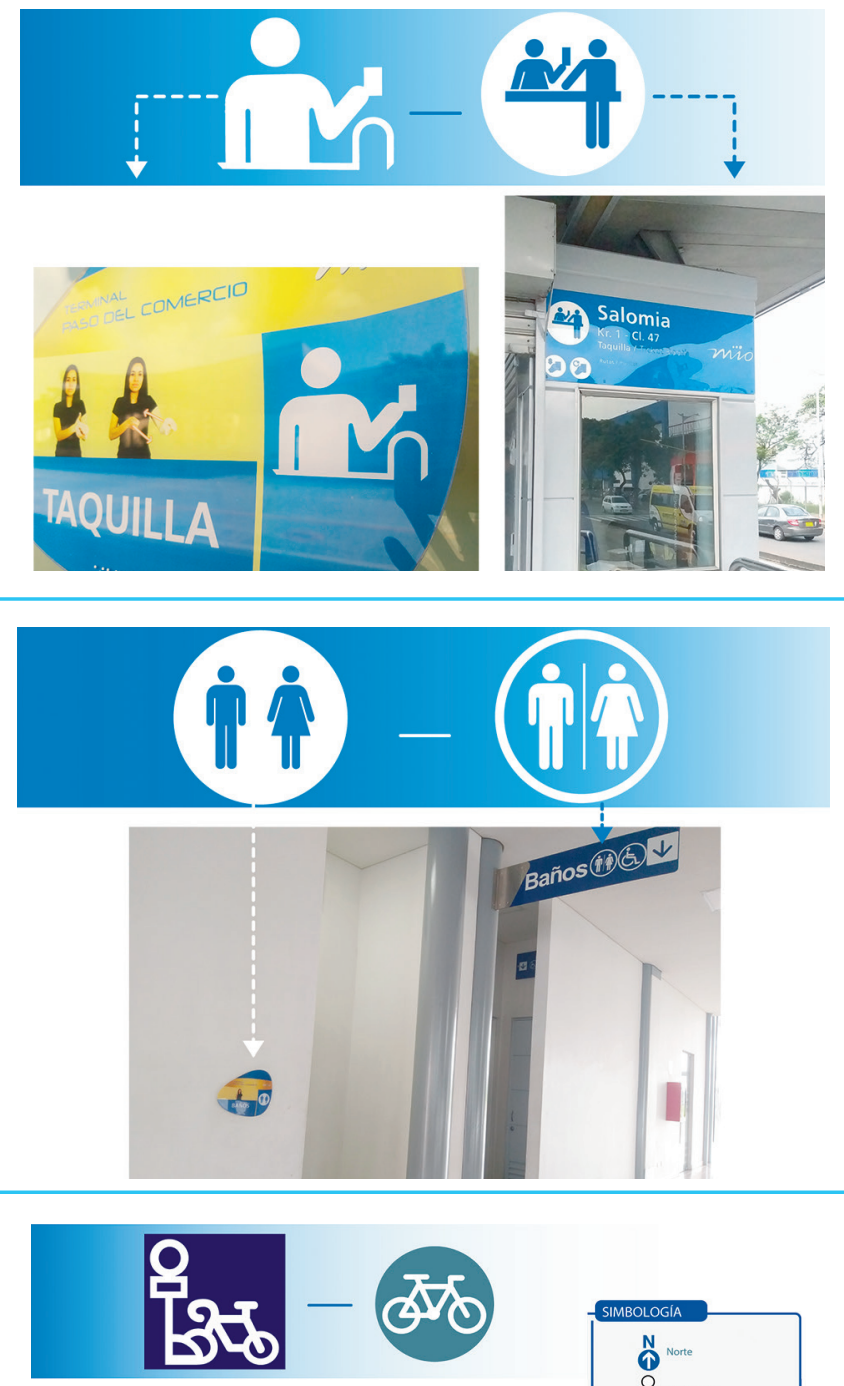

Bici Parqueaderos
en estación y/o Terminal

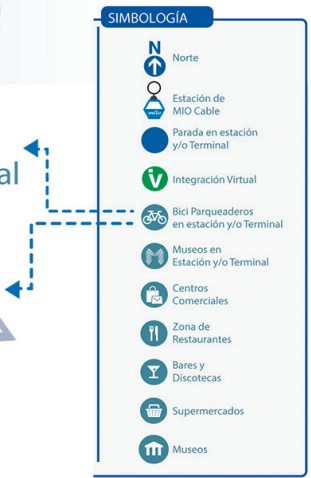

Figura 2. Pictogramas de taquilla. Figura 3. Pictogramas de baños. Figura 4. Pictogramas de cicloparqueadero. Fuente: Gwendy Romo. 
Se planteó un diseño metodológico de tipo descriptivo para indagar contextos sociales que involucran el pensar y actuar de las personas de un grupo (Méndez, 2011). Primero se exploró el contenido y las características de la señalética del sistema de transporte, consignados en sus documentos institucionales. En una segunda etapa se utilizaron cuestionarios para la identificación del problema de investigación en la población adulta mayor (mayores de 60 años), tanto en usuarios del sistema como no usuarios, y confrontar ambas poblaciones en iguales condiciones.

La muestra que respondió el cuestionario 1 fue de 72 adultos mayores, mitad usuarios y mitad no usuarios del sistema de transporte. Se escogió esta cantidad de encuestados teniendo como referencia el dato proporcionado por una de las funcionarias del sistema, quien dirige el programa para adultos mayores (una iniciativa de acercamiento del sistema de transporte a la comunidad), y que informó que el sistema consigue una interacción con 700 adultos mayores en un año.

De este último número se deriva la muestra de esta investigación, equivalente a un 10,28\% de la población (71,96 personas). Se argumenta esta decisión a partir de Bowley (2005), quien afirma que el "muestreo de casos y controles" se utiliza para recoger información de las personas directamente involucradas en las situaciones a indagar, así como de personas que no lo están.

La muestra que respondió el cuestionario 2 fue de ocho usuarios (10\% de la primera muestra), con el objetivo de exponerlos in situ a los pictogramas que tuvieron difícil comprensión en el cuestionario 1.

\section{Proceso de recolección y análisis.}

Los métodos de recolección y análisis de la información que se llevaron a la práctica son principalmente de fuente primaria: la observación y la encuesta, además de que también se tomaron estudios y artículos como fuentes secundarias (Méndez, 2011). Es el caso del estudio de Uribe, Valderrama y Linde (2010), que asocia la vejez con una disminución de la interacción social e incremento del aislamiento, situación que afecta la percepción de los estímulos del mundo exterior. Así mismo, con respecto a la percepción, Pasquel-Padilla (2014) plantea que los adultos mayores tienen más facilidad para comprender formas figurativas y siluetas naturales en tamaño grande. También requieren de contenidos que los conecten con la época vivida en su juventud. En esta misma línea, Sánchez y Pérez (2008) afirman que "los adultos mayores tienen problemas de agudeza y procesamiento visual, problemas de sensibilidad a la iluminación con dificultades de visión en lugares poco iluminados, 
problemas para distinguir colores (especialmente verdes y azules), problemas para enfocar diferentes distancias y déficit en la percepción espacial (percepción del movimiento y la velocidad). Estas dificultades influyen en el tiempo que necesitan para identificar estímulos visuales, y por lo tanto, aumenta el tiempo requerido para resolver tareas perceptivas en comparación con los jóvenes" (p. 5). Finalmente, respecto a las condiciones físicas, Iannicelli (2008) habla en su trabajo de grado de los cambios físicos y motores del adulto mayor que impactan en una motricidad más cuidadosa y lenta, asociada también a la pérdida del oído y la visión.

\section{Observación empírica.}

La observación empírica se complementó con la metodología desarrollada en Uribe (2016), la cual consta de los siguientes pasos:

- Estar en / Reconocer: Ver aquello que todos ven.

- Habitar / Usar: Ver aquello que todos ven, haciendo reflexión de lo visto.

- Vivir / Observar: Ver a profundidad lo que no es fácilmente perceptible.

\footnotetext{
${ }^{2}$ Los funcionarios entrevistados pueden considerarse como "informantes clave", esto es, una "persona que me habla del fenómeno en relación a todo, que tiene amplio conocimiento en relación a todo" (Izquierdo, 2015, p. 1148).
}

\section{Entrevista a funcionarios del sistema.}

Se agendaron citas con funcionarios del sistema de transporte masivo (de la Dirección Comercial y de Servicio al Cliente) para hacerles las siguientes preguntas²:

- ¿Cuántas tarjetas electrónicas de pago personalizadas le han dado a los adultos mayores desde el año 2015 hasta la fecha?

- ¿Cuántos adultos mayores aproximadamente utilizan el servicio?

- ¿Qué información pueden suministrar sobre los adultos mayores como usuarios del sistema? 


\section{Cuestionario 1.}

Se elaboró un cuestionario con los pictogramas del sistema, teniendo como fuente los manuales señaléticos y de normas gráficas institucionales del sistema de transporte analizado (ver Figura 5). Cabe mencionar que en los manuales de señalética se observa una línea gráfica no unificada que, como ya se mencionó, deriva en diversas aplicaciones para la misma información, con dos versiones diferentes del mismo pictograma (ver Figuras 2, 3 y 4).

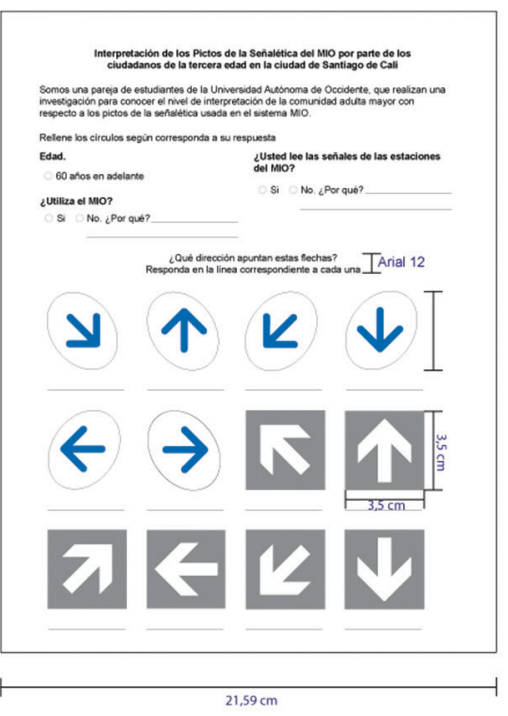

24
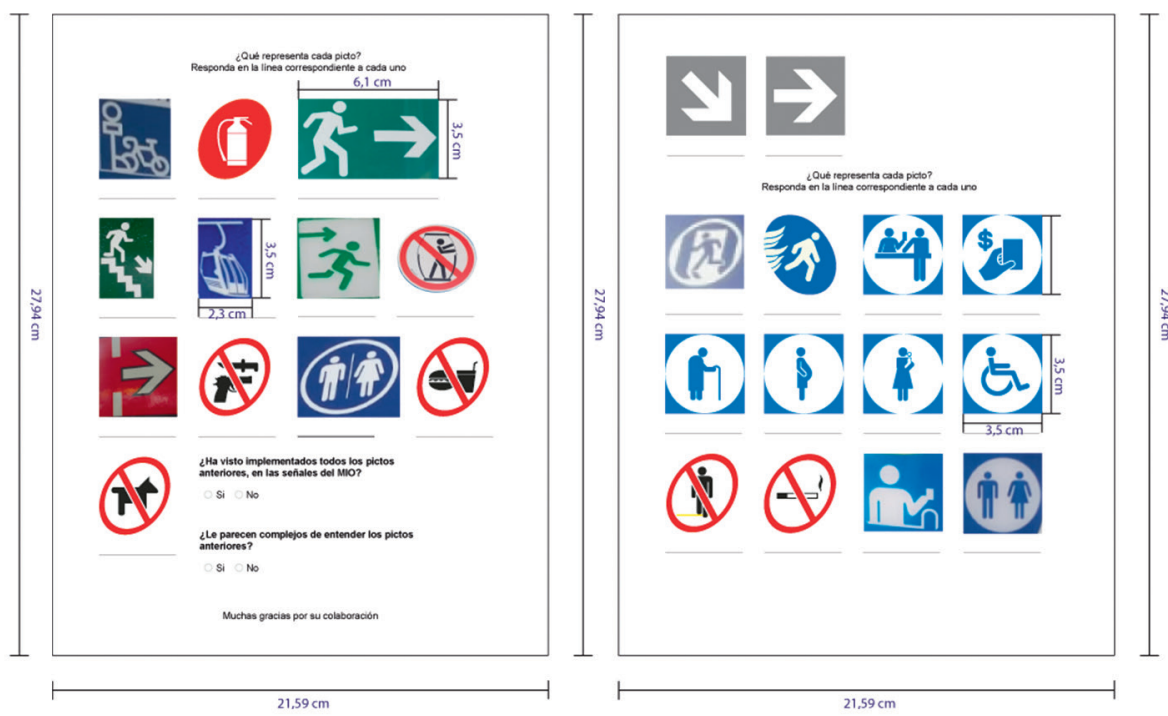

Figura 5. Primer cuestionario, manuales señaléticos y de normas gráficas institucionales del sistema de transporte analizado.

Fuente: Gwendy Romo.

El cuestionario planteado se valida mediante el test de eficacia usado por el Proyecto Guemil (2016), el cual se define como "una iniciativa de pictogramas open source, orientada a representar situaciones de riesgo y emergencia. Permite disponer de un estándar visual para diversas etapas y plataformas de información (antes $\rightarrow$ durante $\rightarrow$ después)" (Guemil project, s.f). El Proyecto Guemil fundamenta su validación mediante la pregunta: ¿Qué representa cada icono?, dando lugar a respuestas abiertas, con el fin de no condicionar a la persona con opciones cerradas, lo que permite obtener con certeza resultados acerca de la comprensión de la población objetivo. 
La aplicación del cuestionario se planteó, en primera instancia, de forma digital; sin embargo, esta estrategia dificultó su acceso al tipo de población que compone la muestra. Por lo tanto, finalmente se aplicó con material impreso, accediendo a la población en iglesias, parques, reuniones y casas. El cuestionario se introduce con la pregunta de si el participante es usuario o no del sistema de transporte, buscando luego indagar la comprensión de pictogramas de emergencia y evacuación (p. ej., extintores, alarmas y salidas en caso de incendio).

\section{Cuestionario 2.}

Para el segundo cuestionario se tomaron fotos de los pictogramas en su contexto actual de ubicación dentro de las terminales y buses. Los pictogramas seleccionados fueron los que tuvieron más bajo nivel de comprensión ${ }^{3}$ en los análisis del cuestionario 1. El cuestionario 2 se imprimió luego en tamaño carta y alta resolución a color para destacar las imágenes (ver Figura 6).

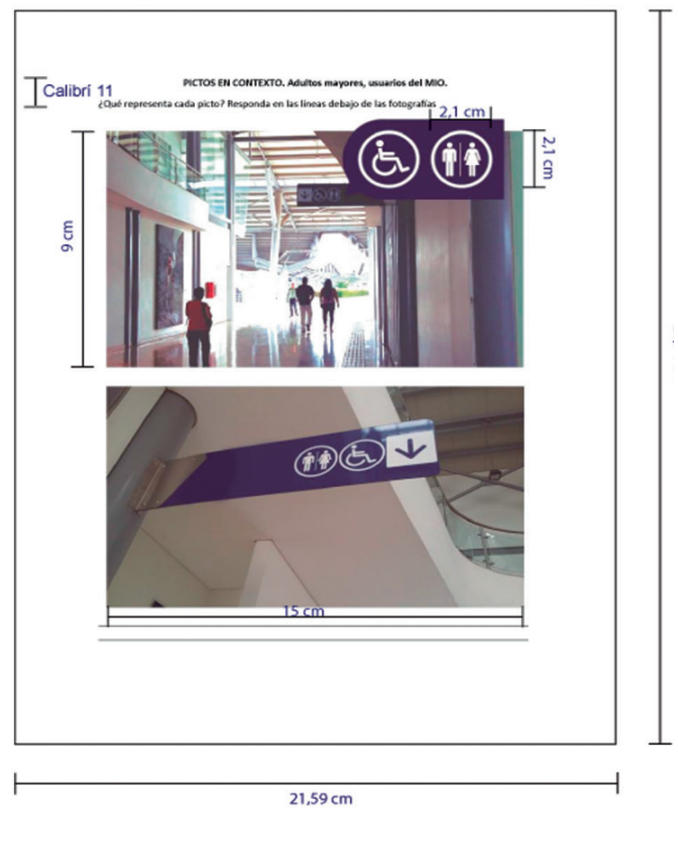

Figura 6. Segundo cuestionario, manuales señaléticos y de normas gráficas institucionales del sistema de transporte analizado. Fuente: Gwendy Romo. 

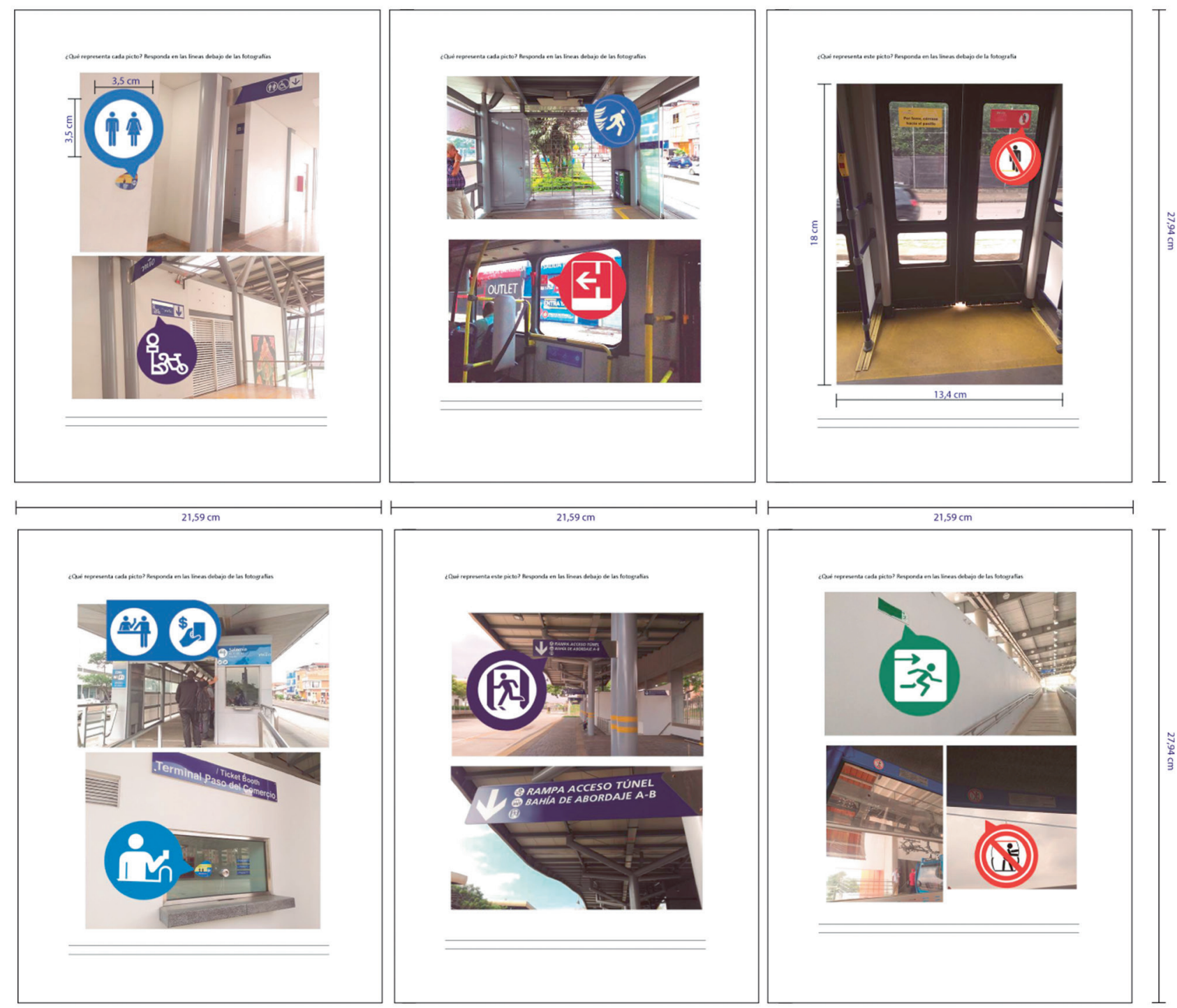

$21.59 \mathrm{~cm}$

$1 \vdash$

$21.59 \mathrm{~cm}$

$1 \longmapsto$

$21.59 \mathrm{~cm}$

Figura 6. Segundo cuestionario, manuales señaléticos y de normas gráficas institucionales del sistema de transporte analizado.

Fuente: Gwendy Romo. 


\section{Criterios de evaluación y tabulación.}

Se realizó una tabulación, tomando como guía la norma ISO 9186-1 "Símbolos gráficos - Métodos de prueba - Parte 1: Método para probar la comprensibilidad" utilizada en el proyecto "Creación y evaluación de pictogramas de señalización" del CEAPAT-Imserso (Centro de Referencia Estatal de Autonomía Personal y Ayudas Técnicas) de Madrid.

En el caso particular del proyecto del CEAPAT (2018) se realizó un "Taller de producción de pictogramas" con 100 personas de diferentes centros participantes, los cuales debían dibujar los pictogramas, vectorizarlos y presentarlos a evaluadores que analizaban las propuestas con sus argumentaciones, para escoger luego la mejor opción. La valoración se regía por el nivel de comprensibilidad del pictograma mediante la escala: "muy comprensible" (100\% de personas lo entendían), "bastante comprensible" (70-99\% de personas lo entendían) y "poco comprensible" (0-69\% de personas lo entendían).

Para la presente investigación se replicaron los criterios de valoración del mismo proyecto, modificando solamente los nombres: "muy comprensible" se denominó como "completa comprensión"; "bastante comprensible" se denominó como "mediana comprensión"; y "poco comprensible" se denominó como "nula comprensión". La valoración fue aplicada a todas las preguntas de cada pictograma para luego graficar la frecuencia de las respuestas.

Para la valoración de las respuestas de los encuestados se tuvo presente:

- La comprensión del concepto representado simbólicamente.

- La forma gráfica utilizada.

- Las asociaciones del concepto que representa.

- La incomprensión del pictograma en cuanto a forma y significado. 
Para definir estos criterios se tuvo en cuenta el trabajo de Beltrán, Durán, Figueroa, Gutiérrez y Zuleta. (2015), en el que se ilustra cómo la percepción y comprensión pictográfica implica reconocer e identificar la forma y el significado asignado, produciendo como resultado un cambio voluntario en la conducta. Otra fuente de apoyo fue la conceptualización acerca de las dimensiones del pictograma que plantea Joan Costa (1989): significado.

Dimensión semántica: Considera las relaciones entre una imagen visual y su

- Dimensión sintáctica: Pone en juego las relaciones de los pictogramas entre ellos.

- Dimensión pragmática: Relaciona el pictograma y su usuario.

- Finalmente, los cuestionarios se imprimieron en formato carta, con impresión láser y a color. Las dimensiones de los pictogramas variaron en ambos cuestionarios.

\section{RESULTADOS}

\section{Entrevista con los funcionarios.}

El funcionario 1 explicó que no es posible conocer el número exacto de usuarios adultos mayores, puesto que las tarjetas electrónicas de pago no son personalizadas y pueden ser usadas por cualquier persona. Además de lo anterior, el sistema no tiene conocimiento de las características específicas del usuario que las usa, a diferencia de las que se personalizan. Esta información podría indicar que para hacer una mejor caracterización de los usuarios y sus necesidades, en el caso del sistema de transporte analizado, se deberían personalizar las tarjetas por grupos etarios.

Igualmente, la funcionaria 2 fue entrevistada con relación al programa que ella dirige con los adultos mayores de la ciudad, el cual desarrolla una serie de actividades entre los meses de marzo a noviembre, donde participan 700 adultos mayores con los cuales se realizan sesiones de ejercicio con la Secretaría del Deporte de la ciudad. Allí también se diseñan programas educativos relacionados con el sistema de transporte, se analizan con ellos criterios de movilidad para su condición física y se les instruye sobre algunos elementos importantes del sistema que tienen vinculación con ellos, como lo son las sillas preferenciales para usuarios en situación vulnerable (embarazadas, ancianos, usuarios con niños pequeños o personas con movilidad reducida). 
Se les enseña el modelo de cultura ciudadana, a lo cual ellos comentan, en palabras de la funcionaria 2:

Aunque ellos son cultos, es decir saben respetar, se ha encontrado que ellos son los que más evaden el tema de la cebra, se pasan el semáforo en rojo, el del peatón se lo pasan en rojo [...] "porque es que tengo afán y necesito llegar". ${ }^{4}$

La funcionaria 2 continúa comentando acerca del respeto de los adultos mayores a la señalética:

Pues el tema ahí, es como por ejemplo si tenemos los flyers de las comunas, se les entrega y ahí están explicados los puntos de recarga más cercanos.

Complementa lo anterior el funcionario 3, vinculado a otro programa del sistema, quien menciona que:

Hay unos trípticos donde están los comportamientos de cultura, [ahí] está incluido lo que te comentaba ella ahora, la prioridad a los adultos mayores, tanto a las personas con capacidades diferenciadas.

El funcionario 3 enfatiza que se distinguen los programas y sus comportamientos según el tipo de población. Para el caso del adulto mayor se omiten temas como el vandalismo, y se refuerza su rol social en la formación de sus nietos y demás miembros de la familia.

Los funcionarios comentaron que a los adultos mayores también se les informa sobre los puntos de recarga de las tarjetas electrónicas de pago en el barrio porque es una pregunta frecuente. También se señaló que se generan espacios para hablar de la memoria histórica y la evolución del transporte en la ciudad, buscando nutrir el conocimiento del sistema con sus saberes y experiencias.

En la entrevista se mencionó además una actividad de socialización para escuchar a la población de adultos mayores, para que compartan sus anécdotas de interacción con el sistema de transporte masivo de manera que se mejoren sus condiciones como usuarios.

${ }^{4}$ Los fragmentos de las entrevistas se presentan con transcripción ortográfica.

Estas entrevistas a los funcionarios evidencian el esfuerzo de la institución por interactuar con su comunidad, enfocando su acción en programas de refuerzo comportamental del usuario. 


\section{Aplicación del cuestionario 1.}

Se observó diferencia en el porcentaje de comprensión completa entre los dos grupos de la muestra (usuarios y no usuarios), siendo los usuarios quienes mayor porcentaje obtuvieron (52\%) (Gráficos 1 y 2).
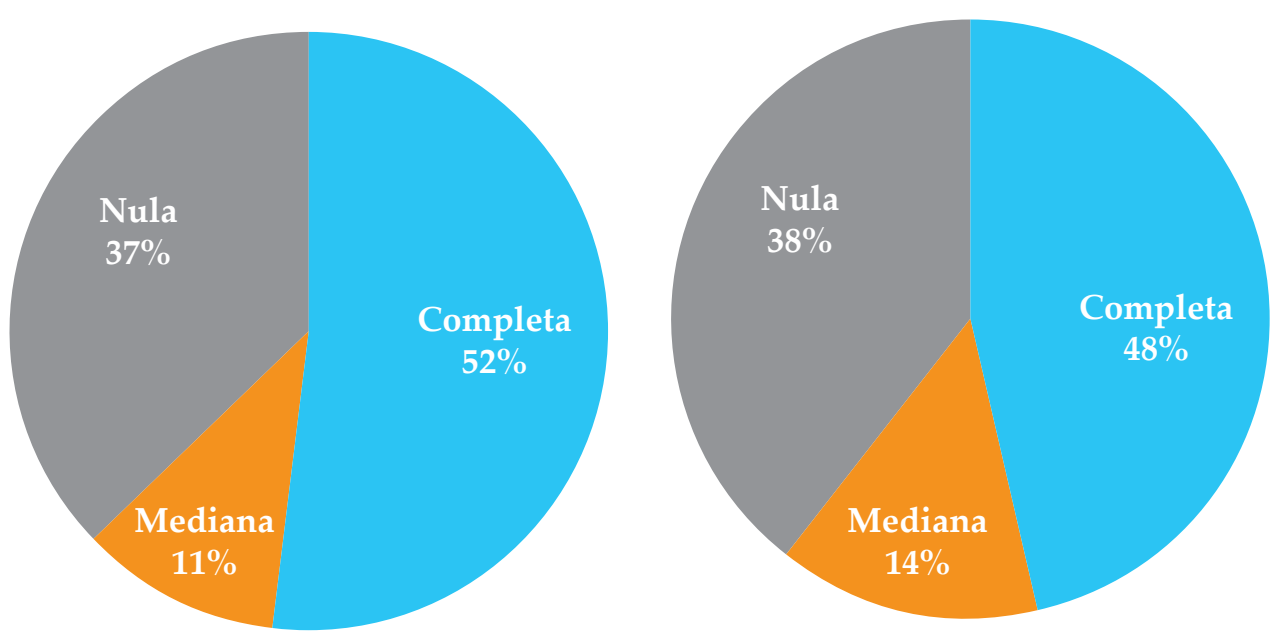

Gráfico 1. Cuestionario 1. Comprensión de los pictogramas por parte de usuarios adultos mayores.

Gráfico 2. Cuestionario 1. Comprensión de los pictogramas por parte de no usuarios adultos mayores. 
Los resultados totales de las respuestas de los encuestados de ambos grupos de la muestra (usuarios y no usuarios) fueron: Completa 50\%, Mediana 13\% y Nula 37\% (Gráfico 3).

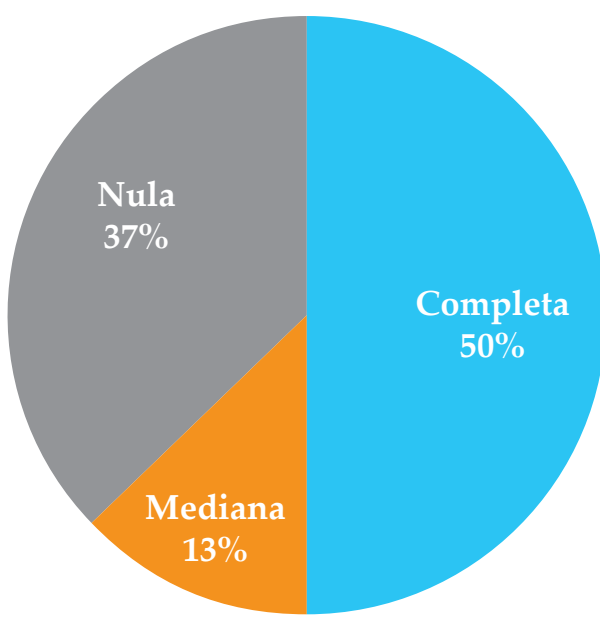

Gráfico 3. Cuestionario 1. Comprensión total de los pictogramas por parte de la muestra completa.

Fuente: Autores.

Como puede verse, la mitad de las respuestas de los adultos mayores encuestados fueron completamente acertadas, con respecto a la comprensión de los pictogramas escogidos de la señalética del sistema. 


\section{Aplicación del cuestionario 2.}

Debido a que en el cuestionario 1 no se tuvo en cuenta la edad de los encuestados, se decidió incluir luego esta variable para analizar como factor influyente en la comprensión de los pictogramas. Como resultado se encontró que el grupo de personas de menor edad de la muestra (encuestados de 62 años), tuvo mayor porcentaje de respuestas con criterio de valoración "comprensión completa" (Gráfico 4).

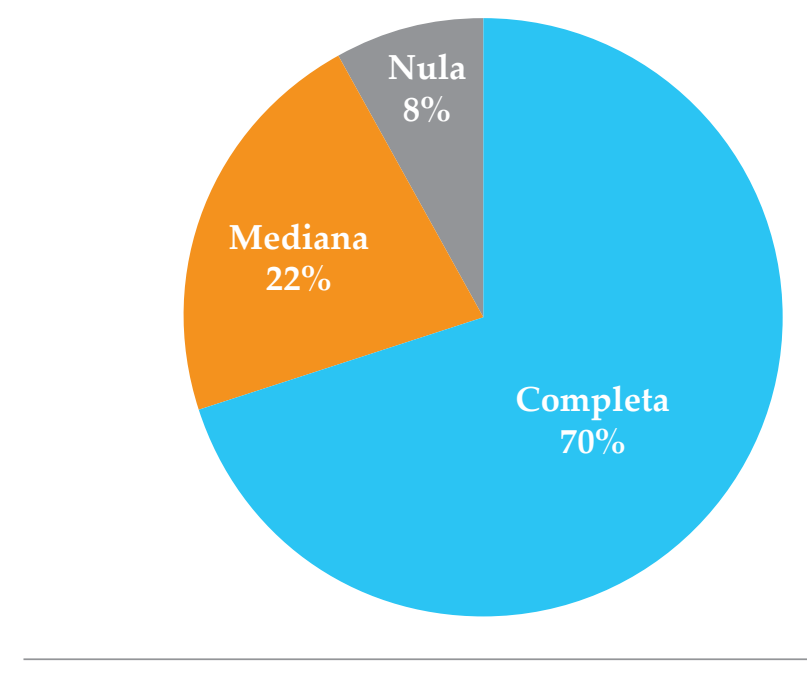

Gráfico 4. Comprensión por parte de personas de 62 años de edad.

Fuente: Autores.

Para el caso del Cuestionario 2, se evidencia que al observar a los pictogramas en su contexto se impacta tanto su percepción como el reconocimiento de la relación entre la imagen visual y su significado, arrojando los siguientes resultados: Completa 62\%, Mediana $18 \%$ y Nula $20 \%$. Los resultados finales de la comprensión del cuestionario 2 se consignan en el gráfico 5, a continuación: 


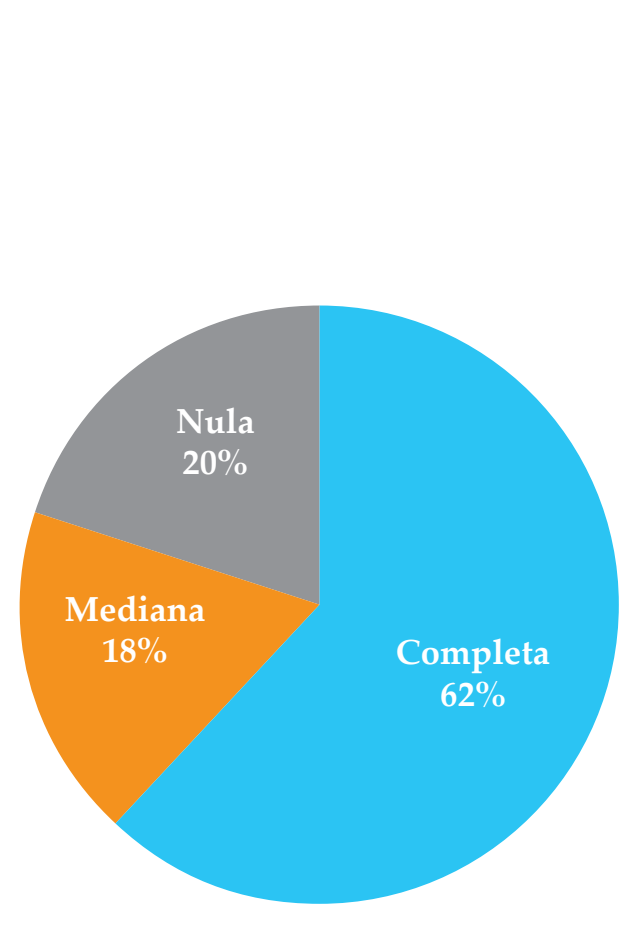

Gráfico 5. Cuestionario 2. Comprensión total de los adultos mayores.

Fuente: Autores.

\section{CONCLUSIONES}

La entidad responsable del funcionamiento del sistema de transporte masivo desarrolla planes enfocados a temas de interacción del usuario con el sistema (ej., uso cívico, cuidado del servicio, procedimientos de uso, acceso a los servicios). Aún no se analiza el grado de comprensión que distintos tipos de usuarios tienen de la señalética, como en el caso de los adultos mayores, quienes, de acuerdo con la revisión de la literatura presentada y los resultados de este estudio, constituyen una población con diversos problemas de salud, que requiere acompañamiento y asistencia ante este tipo de información especializada.

La comprensión de los pictogramas de la señalética por parte de usuarios adultos mayores es baja, debido a la conjunción de distintos factores: visibilidad de la señal, tipo de pictogramas (taquilla, baños, salida, Miocable , salida de emergencia, discapacidad, cicloparqueadero y flechas orientativas), tamaño y legibilidad cuando 
el usuario está expuesto a movimiento. Por consiguiente, la señalética propuesta por el sistema de transporte masivo podría requerir de mayor consistencia: por ejemplo, el caso del pictograma de No pararse dentro del Miocable, con una representación similar a una cubeta de basura (Figura 5), los diferentes pictogramas para ilustrar los conceptos de salidas de emergencia (Figura 6), taquillas (Figura 2), baños (Figura 3) y cicloparqueadero (Figura 4), los cual tienen además doble nominación.
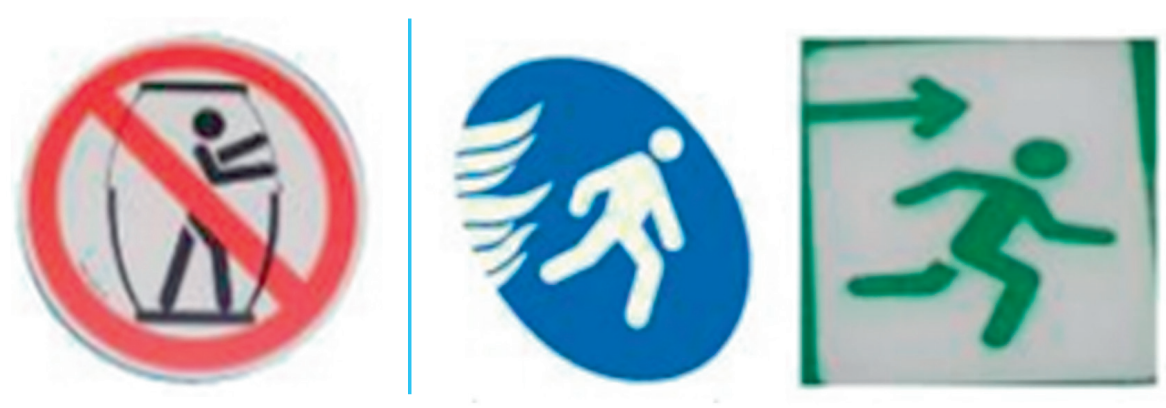

Figura 6. No pararse dentro del Miocable. Figura 7. Señales de emergencia. Fuente: Gwendy Romo.

Sin embargo, habría que estudiar si la confusión en la interpretación de los mensajes por parte de la población adulta mayor se deriva exclusivamente de la señalética, o además podría estar asociada a la educación de los usuarios. De hecho, la entrevista a uno de los funcionarios del sistema de transporte indica que comprender la señalética no implica, necesariamente, aplicarla. Esto refuerza la importancia de diferenciar las dimensiones semántica, sintáctica y pragmática, propuestas por Costa (1989) en el estudio de los pictogramas. 
Teniendo en cuenta las relaciones entre percepción, envejecimiento y toma de decisiones (Sánchez Gil y Pérez Martínez, 2008), podría también decirse que las variables condición física y condición social en los adultos mayores (i.e., personas que viven solas y con rutinas de permanencia en sus casas para distanciarse de dinámicas sociales y de movilidad en la ciudad) influyen en la baja comprensión de un sistema señalético.

En cuanto al color, dentro de la morfología de los pictogramas se ha optado por el uso del azul. Un experimento previo, realizado a 561 personas entre 4 y 71 años, muestra que "en las personas mayores la tendencia a percibir los colores en tonalidades más oscuras se asocia con la dificultad para distinguir tonalidades que difieren en su contenido cromático de azul, debido al oscurecimiento de la córnea y el cristalino, fenómeno que se produce a partir de los 30 años" (Correa et al., 2007, p. 12). Por consiguiente, el color azul puede generar inconvenientes de legibilidad bajo ciertas circunstancias (en la noche o con lluvia). Sin embargo, esta condición no se presentó como crítica dentro de la encuesta, tal vez porque los pictogramas evaluados están ubicados en la zona preferencial del bus articulado, la cual ellos frecuentan (Figura 7).

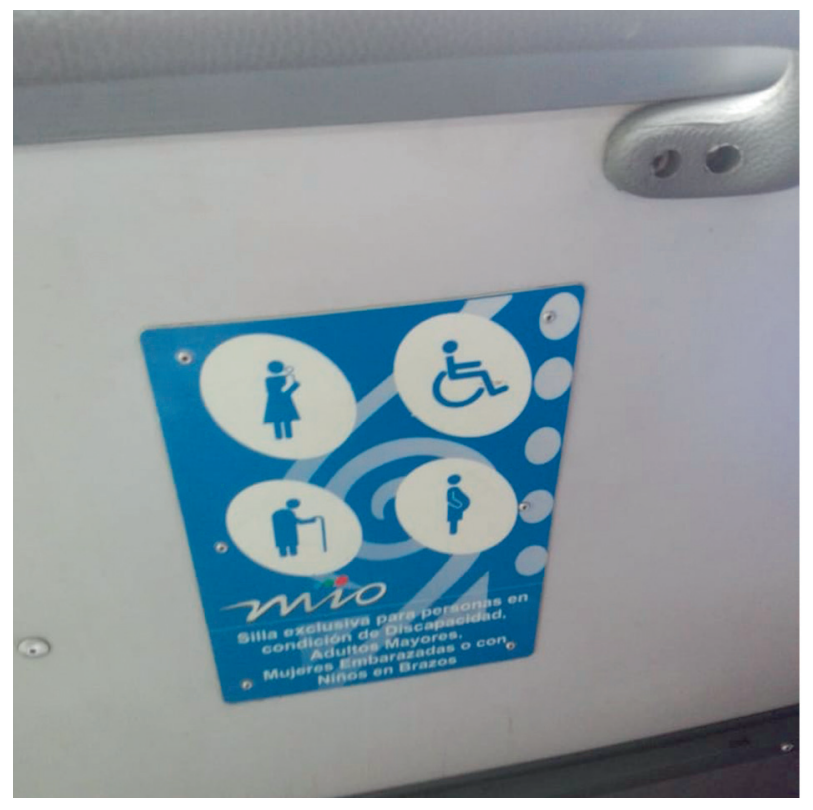

Figura 8. Pictogramas de la zona preferencial. Fuente: Gwendy Romo. 
Las condiciones espacio-temporales son fundamentales para el desempeño perceptual y comprensivo de una señal por parte de los usuarios. Esto se corroboró como resultado en el cuestionario 2, basado en pictogramas que obtuvieron baja comprensión en el cuestionario 1, pero presentados en su contexto de ubicación. Ofrecer mayor información de contexto facilitó evaluar la funcionalidad del pictograma, al integrar el usuario condiciones que influyen la comprensión, como: espacios restringidos o amplios, aglomeración de personas, baja luminosidad o distancia de la señal.

Los resultados finales indican que el criterio de valoración de "completa comprensión" de la señalética fue de un 50\% por parte todos de los usuarios de la muestra. Esto puede leerse como un resultado bajo sobre la funcionalidad de la señalética, según el proyecto CEAPAT (2018), en el cual la calificación de "muy comprensible", correspondiente en este estudio con una "completa comprensión", ha de superar una calificación igual o superior al 70\%. A partir de lo anterior, se infiere que la señalética debe ser revisada, enfocándose en particularidades de su población de usuarios. Dichas evaluaciones deben tener en cuenta las características culturales e históricas de los usuarios como indicadores de gestión en el análisis del sistema señalético. Por ejemplo, para el caso de la población definida en este estudio debería tenerse en cuenta la relación entre el esfuerzo cognitivo y la morfología de la información gráfica.

\section{RECOMENDACIONES}

Brindar una mejor experiencia al usuario, para lo cual la señalética del sistema de transporte masivo debería unificar su línea gráfica. Por ejemplo, el pictograma "cicloparqueadero" se asocia a una señal de tránsito y no es interpretada de manera adecuada (Figura 8); podría optarse por la continuidad de la línea gráfica de modo que permita dar relevancia al objeto donde se recuestan las bicicletas, para evocar el lugar y asociarlo con el servicio. De esta manera fue comprendido por los participantes del cuestionario 2 . 


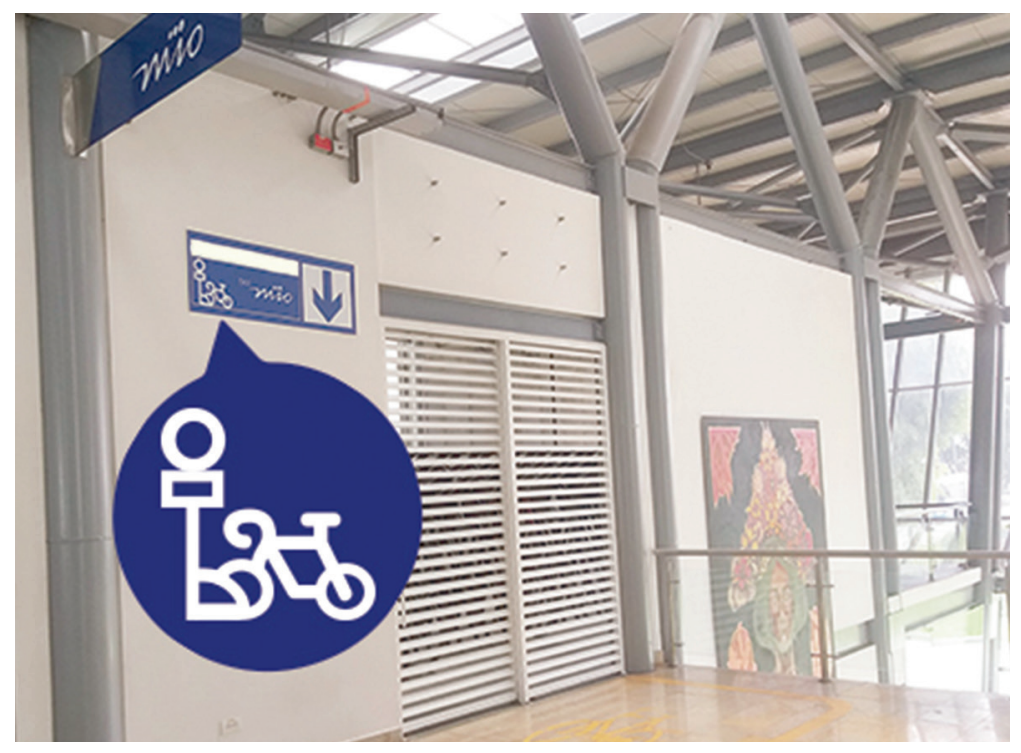

Figura 9. Pictograma de cicloparqueadero.

Fuente: Gwendy Romo.

Nutrir el programa de adultos mayores con salidas a las paradas del sistema (estaciones y terminales) para explicar a los usuarios in situ la función de la señalética, y poner en práctica lo enseñado mediante la simulación de un recorrido.

Replantear el diseño actual de los pictogramas menos comprendidos por los usuarios de la muestra. Priorizar el rediseño de los pictogramas de emergencia, dada su importancia. Para esto, reutilizar aspectos de los pictogramas de emergencia mejor comprendidos: usar la figura humana; indicar la dirección a tomar; describir la acción a ejecutar (p. ej., romper la ventana).

Realizar estudios que incluyan un análisis acerca del tiempo requerido para la comprensión de los pictogramas, teniendo en cuenta las relaciones establecidas entre la percepción, el envejecimiento y la toma de decisiones (cfr. Sánchez Gil y Pérez Martínez, 2008).

\section{Agradecimientos}

Esta investigación fue posible gracias a los participantes voluntarios de este estudio. Se agradecen, en particular, los comentarios y ediciones realizados durante la revisión ciega de pares. 


\section{REFERENCIAS BIBLIOGRÁFICAS}

Beltrán, F., Durán, M., Figueroa, C., Gutiérrez, M. \& Zuleta, P. (2015). Percepción de pictogramas de señalización. En Beltrán, F. y Durán, M. (Eds.). Pictogramas de Señalización. Miradas Interdisciplinarias (pp. 57-81). Bogotá: Universidad Jorge Tadeo Lozano.

Bowley, A. (2005). «Muestreo» (An elementary manual of statistics). EMPIRIA. Revista de Metodología de las Ciencias Sociales, (10), 213-224. Recuperado de http://www.redalyc.org/articulo.oa?id=297123998008

Cardona, J. \& Carvajal, D. (22 de mayo de 2015). 400 tarjetas del MIO fueron entregadas a los adultos mayores. Alcaldía Santiago de Cali. Recuperado de http://www.cali.gov.co/bienestar/publicaciones/108993/ tarjetas_del_mio_fueron_entregadas_a_los_adultos_mayores/.

CEAPAT (2018). Creación y evaluación de pictogramas de señalización. Recuperado de https://ceapat.imserso. es/InterPresent2/groups/imserso/documents/binario/crepictogramas2018.pdf.

Correa, V., Estupiñán, L., García, Z., Jiménez, O., Prada, L., Rojas, A., ... Cristancho, E. (2007). Percepción visual del rango de color: diferencias entre género y edad. Revista Med, 15 (1), 7-14. Recuperado de http://www.redalyc.org/articulo.oa?id=91015102

Costa, J. (1989). Señalética. Barcelona: Ceac. Recuperado de https://es.scribd.com/doc/34740719/ senaletica-de-la-senalizacion-al-diseno-de-programas-Joan-Costa.

Guemil (2016). Proyecto Guemil. Recuperado de http://www.guemil.info/. 
Iannicelli, F. (2008). Diseño inclusivo aplicado al Pami [Trabajo de grado]. Buenos Aires: Facultad de Diseño y Comunicación, Universidad de Palermo. Recuperado de: https://www.palermo.edu/dyc/ pgraduacion/archivos_bajada/mejores_pg/iannicelli.pdf

Izquierdo, G. M. (2015). Informantes y muestreo en investigación cualitativa. Investigaciones Andina, 17(30), 1148-1150. Recuperado de https://www.redalyc.org/pdf/2390/239035878001.pdf

Lámbarry, F., Rivas, L. \& Trujillo, M. (2013) Desarrollo de una escala de medición de la percepción en la calidad del servicio en los sistemas de autobuses de tránsito rápido a partir del Metrobús de la Ciudad de México. Innovar, 23(50), 79-92. Recuperado de https://www.redalyc.org/articulo. oa?id=81828692007

Marqués, L. (2009). La interpretación de los pictogramas utilizados en la dispensación del medicamento de acuerdo con la perspectiva de personas con diferentes niveles de educación. Research Gate. 3-6. Recuperado de https://www.researchgate.net/publication/264543287

Méndez, C. (2011). Metodología. México DF: Limusa SA. Recuperado de https:/es.scribd.com/ document/324262554/METODOLOGIA-DE-LA-INVESTIGACION-CARLOS-MENDEZ-1-pdf

Ojeda-Díaz, A. J. (2018). Impacto de los sistemas BRT en percepciones de cambios en el bienestar social y el entorno: caso de Barranquilla, Colombia [Tesis de maestría]. Universidad del Norte. Barranquilla, Colombia. Recuperado de http://manglar.uninorte.edu.co/bitstream/handle/10584/8183/1140878350. pdf?sequence $=1 \&$ isAllowed $=\mathrm{y}$

Pasquel-Padilla, S. (2014, Julio 21). La percepción visual en el adulto mayor. Herramienta para comunicadores visuales. ISUU. Recuperado de: https://issuu.com/titiago/docs/la_percepci_n_visual_en_adultos_ma

Sánchez Gil, I., \& Pérez Martínez, V. (2008). El funcionamiento cognitivo en la vejez: atención y percepción en el adulto mayor. Revista Cubana de Medicina General Integral, (24)2, 2-6. Recuperado de http:// scielo.sld.cu/scielo.php?script=sci_arttext\&pid=S0864-21252008000200011\&lng=es\&tlng=es.

Uribe, M. (2016). Diseño de información: una herramienta para el uso y apropiación del transporte público. Cali: Editorial de la Universidad Autónoma de Occidente.

Uribe, A. F. R., Valderrama, L. J. V., \& Linde, J. M. M. (2010). Intervención psicológica en adultos mayores. Psicología desde el Caribe, (25), 246-258. Recuperado de http://www.redalyc.org/articulo. oa?id=21315106011

Vega, O., Rivera-Rodríguez, H., \& Malaver, N. (2017). Contrastación entre expectativas y percepción de la calidad de servicio del sistema de transporte público de autobuses en Bogotá. Revista Espacios, 38(43), 3-15. Recuperado de https://www.revistaespacios.com/a17v38n43/a17v38n43p03.pdf 